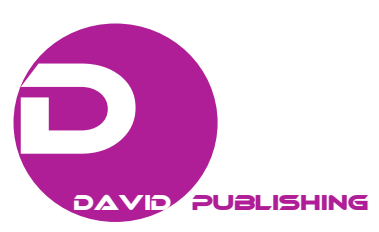

\title{
Using Surface Splines for GIS- ready Imagery
}

\author{
Ruan Wei \\ Tong Ji University, Shanghai 200092, China
}

\begin{abstract}
High resolution data have become an important source of data, before they can be integrating into a GIS database. It requires processing for ortho-rectification to generate image map with high accuracy and low cost. Using surface splines interpolation for rectification is quite different with traditional grid method in photogrammetric. To introduce surface splines is important. In fact the actual name is mechanical surface splines because it must add mechanical conditions to form the formula. The main advantages of the surface splines are that the coordinate of the known points are not located in a rectangular array and the function may be differentiated in find slopes. Surface splines are a mathematical tool to interpolate a function of two variables. It bases upon small deflection equation of an infinite plate, it originally developed for interpolation wing deflection of aircraft 1972 by Harderaed and Desmarais contributed. An example map size is beyond the custom. Only five control points for rectification $(60 \mathrm{~cm} \times 200 \mathrm{~cm})$ if the number of control points is increasing also the map size increasing large and operating easy. The other UAV in civilian world gives low cost. Obvious aerial imaging using surface splines in cartographic is a tool for supporting geospatial decisions.
\end{abstract}

Key words: Surface splines, rectification, interpolation, two variable function, imagery.

\section{General Background}

In the field of surveying the view points of common knowledge for interpolation could write the equation observation as

$$
\mathrm{L}=\mathrm{AX}+\mathrm{BT}+\mathrm{n} \text { (noise) }
$$

Where $\mathrm{A}$ is given $\mathrm{q} \times \mathrm{p}$ matrix expressing the effect of the parameters $\mathrm{X}$ on the observation $\mathrm{L}$.

$\mathrm{B}$ represents linear operator and comprises the $\mathrm{q}$ linear functional, q-vector.

Otherwise surface splines gives the same math type (Eq. (4)), so in photogrammetric using surface splines naturally.

In Eq. (4), $\sum_{i=1}^{N} F_{i} r_{i}^{2} \operatorname{In} r_{i}^{2}$ also gets the character of the reproducing kernel $\mathrm{k}(\mathrm{x}, \mathrm{y})$ in Hilbert space, which can ensure the receiving of minimal norm solution for interpolation in photogrammetric.

As the famous professor Karl Kraus said that mathematical model 4 has determined the trend function and the part treated with least squares interpolation together which solved the problem in an

Corresponding author: Ruan Wei, professor, research fields: surveying, GIS, filter, computational mechanics and big data analysis. elegant way.

\section{Mathematical Analysis}

The formula derivation goes very strict: as following:

As we know that a linear spline is the solution of an equation based upon a small deflection of an infinite beam.

The surface splines is the deformation of an infinite plate bending only.

The differential equation is relating bending deflection and the load of the plate is

$$
\text { D } \nabla 4 \mathrm{~W}=\mathrm{P}
$$

$\mathrm{D}=$ flexural rigidity of the plate;

$\mathrm{W}(\mathrm{x}, \mathrm{y})=$ lateral deflection;

$\mathrm{P}=$ lateral load.

Deflections are specified at $\mathrm{N}$ independent points (xi, Yi) $\mathrm{i}=1 \ldots \mathrm{N}$. Here it needs to know the point loads $\mathrm{Pi}$ at these $\mathrm{N}$ points. When the load is determined, the deflection will be determined too.

For example, if we want to know the symmetrical deflection of the origin point

when the load is $\mathrm{P}$,

use the Eq. (1) 
where

$$
\begin{aligned}
& X=r \cos \theta \\
& Y=r \sin \theta
\end{aligned}
$$

And we get

$$
\mathrm{W}(\mathrm{r})=\mathrm{A}+\mathrm{Br} 2+(\mathrm{P} / 16 \pi \mathrm{D}) \mathrm{r} 2 \cdot \ln \mathrm{r} 2
$$

In Eq. (2), A, B are undetermined coefficients while $\mathrm{P}$ is point load.

If continue, the derivation will also add two conditions;

Deflection is symmetrical at the points with load.

The surface splines will be flat if far away from the applied loads.

The deflection of the whole splines is the summation of Eq. (2)

$$
\mathrm{W}(\mathrm{x}, \mathrm{y})=\sum \mathrm{A}+\mathrm{Br} 2+(\mathrm{P} / 16 \pi \mathrm{D}) \mathrm{r} 2 . \ln \mathrm{r} 2
$$

And after deduced and combined, we get

$$
\mathrm{W}(\mathrm{x}, \mathrm{y})=\mathrm{A}+\mathrm{Bx}+\mathrm{Cy}+\sum_{i=1}^{N} F_{i} r_{i}^{2} \operatorname{In} r_{i}^{2}
$$

Here ri2 $=(x-x i) 2+(y-y i) 2$, using Eq. (4) needs to solve $\mathrm{N}+3$ unknown quantities.

Here $\mathrm{N}=5$,

so add 3 equilibrium equations and then form 8 linear equations, and get the final answer.

In this way which can correct the image point by point while avoid its translating and rotating, that is due to the function of the equilibrium equations.

Eq. (5) is an equilibrium equation.

$$
\sum F i=\sum X \text { i } F_{i}=\sum Y i F i=0
$$

\section{Function of the Software}

This software can make image map $50 \mathrm{~cm} \times 50 \mathrm{~cm}$ from high resolution data based on five control points. The name is "five points method". It's easy to operate, anyone who can use PC works with it smoothly: there's no special skill for operation and the data to enter are little. It's easy to find an appropriate PC, for any ordinary PC can do the job.

The processing steps are as following:

(1) Scan image into PC;

(2) Enter the coordinate of the control points;

(3) Identify the control points on the image (recognition of control points);

(4) Supply mapping scale;

(5) Rectifications.

\section{Example}

The map size is large beyond the custom size without any splices and modification. Using five control points for rectification is from high resolution data to generate 1:500.

\section{Conclusion}

Surface splines is useful in photogrammetric and survey, it depends upon the solution of linear equations.

The closed form solution involves no function more than logarithms, and is easy coded.

Other using of interpolation was treating the residuals of block strip triangulation in photogrammetric, which also gets successful.

Now, particular using surface splines in cartographic is a nice tool for supporting agriculture, forest, urban, geospatial decisions.

\section{References}

[1] Robert L. H., and Robert N. D. 1972. "Interpolation Using Surface Spline.” Aircraft 9 (2): 189-91.

[2] Wang, Z. Z. 1986. Continuation of Concepts for Photogramme, 184-7. 
Image map

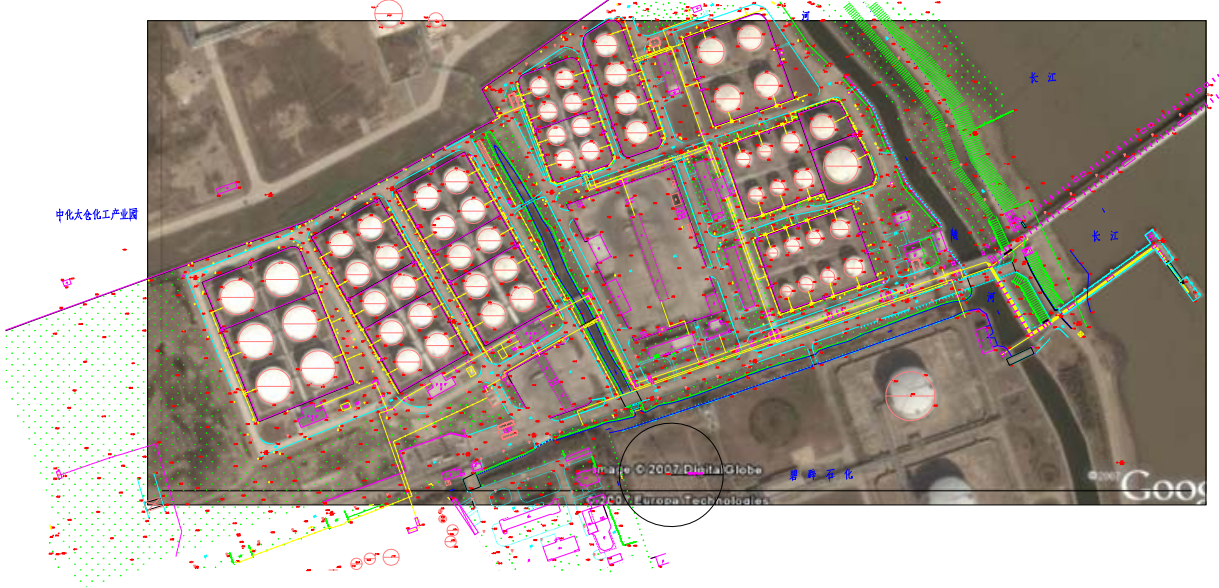

